\title{
AN ATYPICAL MANIFESTATION OF CAT SCRATCH DISEASE: PARINAUD'S OCULOGLANDULAR SYNDROME
}

M.Oikonomakou ${ }^{1}$, K.Kourelis ${ }^{2}$, E.Koufoglou ${ }^{1}$, E.Panoutsou ${ }^{1}$

1. Pediatric department, General Children Hospital of Patras, Greece

2. ENT department, General Children Hospital of Patras, Greece

\section{Background}

Cat scratch disease (CSD) is a common benign disease in children, caused mainly by Bartonella henselae. Parinaud's oculoglandular syndrome appears to be the most common ocular complication of CSD, affecting approximately $5 \%$ of symptomatic patients. This syndrome occurs when $\mathrm{B}$. henselae bacilli are inoculated onto the conjunctiva of the patient, resulting in a conjunctival disease characterized by a unilateral granulomatous follicular conjunctivitis associated with ipsilateral regional lymphadenopathy.

\section{Case presentation}

A 13 old-girl presented with a 2-week history of swelling of the right parotid region. The patient was afebrile,with no other symptoms.She had received per os treatment with amoxicillin/clavulanic acid with no signs of improvement.Physical examination revealed unilateral mild papular lesions of the lower eyelid of the right eye that had been presented 17 days before admission (fig.1). There was, also, 3x3 $\mathrm{cm}$ swelling present just anterior to the right tragus. The swelling was firm and attached to the skin. There was no local tenderness. Ultrasound showed a round hypoechoic intraparotid node measuring $1 \times 1.5 \mathrm{~cm}$ in diameter(fig.2). These features were considered virtually diagnostic of CSD.On subsequent questioning there was clear history of being scratched by their recently acquired kitten.Diagnosis was confirmed by serological tests. The patient received per os clarithromycin with uncomplicated clinical course and full recovery one month later.

\section{Learning points/conclusion}

Slow-growing painless neck masses in children are generally alarming and physicians frequently rush into biopsy or resection to exclude a neoplastic etiology. However, apart from the cervical lymphadenopathy itself, history taking and physical exam should always look for a portal of entry of an infectious agent in the head and neck region, leading to a much less invasive diagnostic and therapeutic management

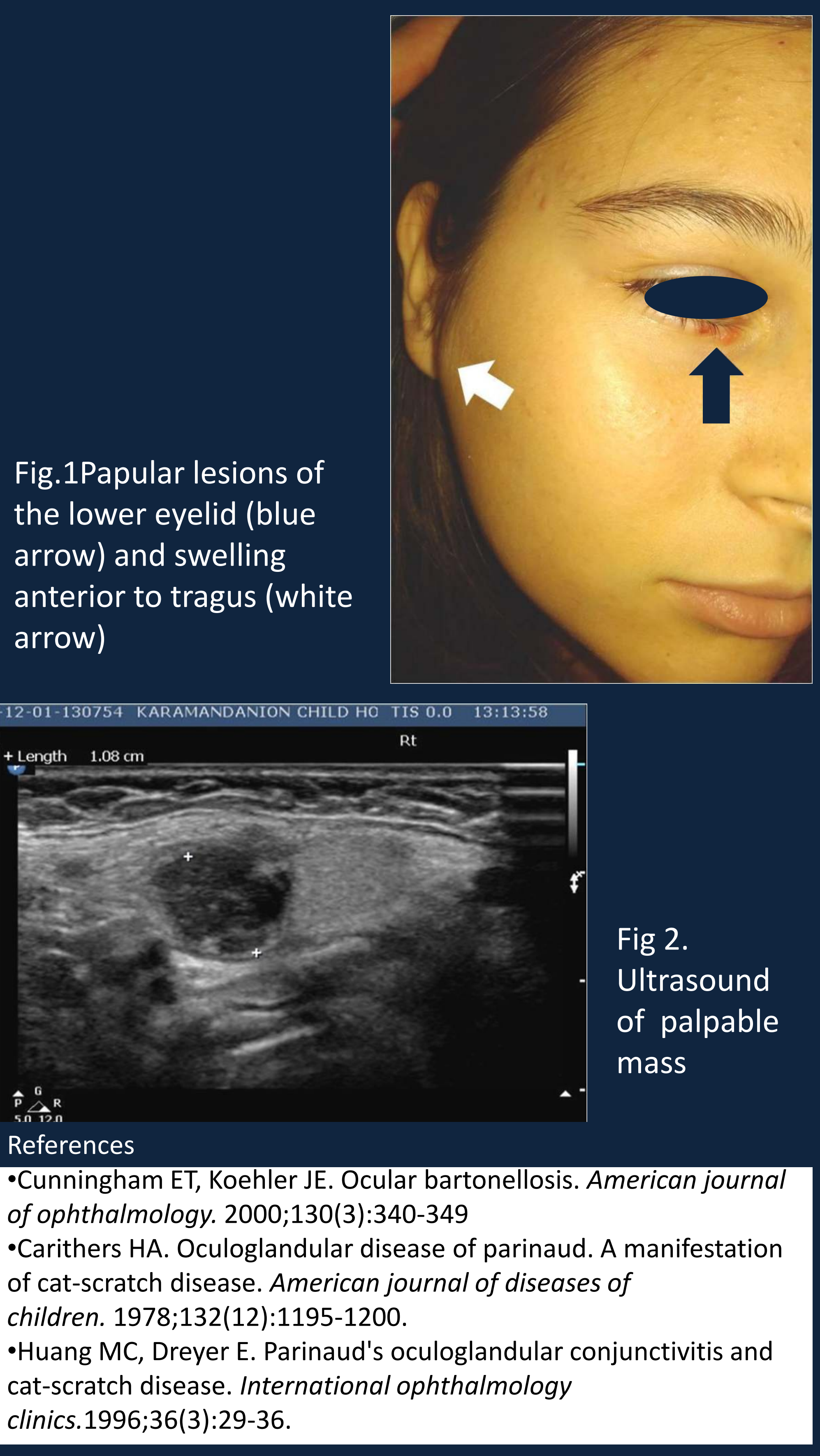

